Revista Educación 25(1): 53-66, 2001

\title{
PERCEPCIONES SOBRE LA FORMACIÓN DOCENTE Y SU POSIBLE ARTICULACIÓN CON LA ENSEÑANZA DE LA MATEMÁTICA: UN ESTUDIO DE CASOS
}

\author{
Tania Elena Moreira Mora
}

\begin{abstract}
Resumen: En este artículo se reportan los hallazgos preliminares de un estudio de las percepciones de los profesores de secundaria, acerca de su formación en el campo de la Matemática y su posible enlace con la articulación metodológica y con la actitud de los estudiantes hacia esta materia en la región educativa Huetar Atlántica durante el año de 1996.

Se realizó un estudio de casos con los cinco profesores que concluyeron su formación profesional en el Plan Regular de la Universidad de Costa Rica, en la sede regional de Limón. Este plan se inició en 1992 con una duración de tres años y se otorgó el grado de profesorado.
\end{abstract}

\section{Introducción}

En el campo de la enseñanza de la Matemática existe una gran deficiencia en los conocimientos y destrezas cognoscitivas de los estudiantes y las estudiantes demostrada en el rendimiento académico, específicamente en las pruebas nacionales de Bachillerato en Educación Media, cuyo índice de reprobación fue el más alto de todas las asignaturas evaluadas $(35,21 \%)$. Esta situación se agudiza aún más en la región Huetar Atlántica, con sólo un 42\% de aprobación (MEP: 1995).

Sumado a esta problemática está la carencia de docentes con formación profesional para la enseñanza de la Matemática en III ciclo y educación diversificada. En el caso particular de la provincia de Limón el 90\% de los profesores son aspirantes y autorizados (CONARE, 1991). Como una posible solución a esta carencia en 1992 PROMECE (Programa para el Mejoramiento de la Calidad de la Educación del Ministerio de Educación Pública) inició diversos programas, entre ellos el de formación docente en el Componente de Desarrollo Profesional. Para su puesta en marcha se estableció un convenio con las universidades estatales.

El propósito cardinal de este programa, instituido por medio del Convenio MEP - Universidades Estatales, fue reducir el número de docentes de I, II y III ciclos sin preparación formal, cuyas metas establecían lo siguiente: 
- Diplomar en un período de cinco años a 3333 aspirantes de primer y segundo ciclos mediante cursos de dos años de duración.

- Preparar en cinco años a 1.188 docentes de tercer ciclo mediante cursos de tres años en un campo específico.

- Preparar a 700 docentes del ciclo III que tienen varios años de experiencia y algún tipo de educación de mayor nivel pero carecen de formación pedagógica (autorizados). (PROMECE, 1995).

Para la consecución de estos objetivos se planteó la necesidad de impartir cursos de certificación, de actualización y de perfeccionamiento. Los cursos de certificación se impartirían en dos modalidades. La primera tenía, a su vez, dos vertientes: una dirigida a certificar 3.333 docentes aspirantes (sin estudios universitarios) de I y II ciclo por medio de un programa de formación de dos años de duración y la otra dirigida a certificar 1.188 docentes (también aspirantes) de III ciclo, con un programa de tres años de duración, con el que se graduarían con el título de profesor. La segunda modalidad de los cursos de certificación estuvo dirigida a 700 docentes del III ciclo con alguna experiencia y alguna educación superior (llamados autorizados), pero sin formación pedagógica. Se estimó que con un programa de dos años (con adecuaciones que responderían a sus conocimientos particulares) se graduarían también como profesores.

En la Universidad de Costa Rica, particularmente en la sede regional de Limón, el convenio tuvo dos modalidades en el campo de la Matemática. Una de ellas fue el Plan Regular con una duración de tres años, en el que se obtenía el título de profesor, otorgándose la categoría de MT 3, según la Ley de la Carrera Docente. La otra es el Plan de Formación de Profesores en Servicio, con dos años de duración, y al finalizar se ubicaban dentro de la categoría MT 2, según la Ley de Carre- ra Docente. En este estudio se escogió el primer plan porque los participantes concluyeron su formación profesional con el grado de profesorado.

\section{Propósitos generales}

En el estudio se pretendió un acercamiento inicial a las percepciones de los profesores de Matemática graduados sobre su formación docente y la posible vinculación con sus articulaciones metodológicas y, consecuentemente, con las actitudes de los estudiantes.

Asimismo, se procuró identificar las creencias que moldearon la actitud hacia la Matemática en aquellos estudiantes que interactuaban con los profesores graduados.

\section{Fundamentos teóricos}

La literatura consultada se centró en los tópicos de rendimiento y actitud de los estudiantes y las estudiantes hacia la materia, así como en la formación profesional del docente.

Los diversos estudios realizados en la dimensión del rendimiento académico, a criterio de Pereda y Mazaredo (1988) fueron poco fiables cuando se basaron en la promoción escolar, debido a las diferencias en el nivel de exigencia entre una institución y otra por la heterogeneidad de los currícula en las aulas. Además, señaló que el rendimiento real es inferior al reflejado por las estadísticas oficiales de promoción. En este sentido, en otro estudio, se destacó que las divergencias en las calificaciones de una prueba aplicada para medir un curriculum básico se justifican por el tratamiento metodológico y didáctico (Apraiz, J. et al: 1988).

En este estudio la noción de rendimiento académico se enfocó hacia la perspectiva del desarrollo de estrategias cognoscitivas, definidas por Wittrock (1990:544) como "la conciencia o percepción de la enseñanza, la atención que se le presta, la 
motivación para aprender y la capacidad para generar relaciones entre el conocimiento y la experiencia, por una parte, y los materiales o conceptos que deben aprenderse, por la otra".

Asimismo, se retomó el concepto de actitud planteado por Kerlinger (1994:514).

...una predisposición organizada para pensar, sentir, percibir y comportarse hacia un objeto de referencia o cognoscitivo. Es una estructura permanente de creencias que predispone al individuo a comportarse de manera selectiva hacia las referencias de actitudes. El referente es una categoría, una clase o conjunto de fenómenos: objetos físicos, eventos, comportamientos o incluso constructos.

En el proceso de formación profesional del docente en el campo de la enseñanza de la Matemática, se subrayó en una investigación realizada en Estados Unidos (Contreras, 1995) que éste no es significativo en el desempeño profesional, puesto que a excepción de la práctica docente y unos cursos, su formación fue abstracta e idealista sin enlazarla con la realidad de su quehacer diario en el aula. Además, se percibió como una superposición de dos partes: la formación en Matemáticas y la formación en educación.

En el proceso educativo, señala Contreras que el aprendizaje se convirtió en un proceso mecánico y memorístico de un conjunto de algoritmos y procedimientos presentados a los educandos como un producto acabado. De ahí que, según este estudio, el estudiante se caracterizó por ser pasivo en la adquisición y construcción del conocimiento, puesto que las actividades del estudiante consistían en escuchar, observar y tomar notas de lo que el profesor hacía en la pizarra y posteriormente repetir los procedimientos hasta mecanizarlos o memorizarlos.

El rol de los profesores de Matemática se identificó con la actividades que desarrollaban y con los significados que estas tenían para los participantes en el proceso educativo. Según este estudio, fueron cuatro categorías básicas:
- $\quad$ vigilante o encargado del cuido de los adolescentes;

- $\quad$ entrenador de exámenes;

- mediador;

- innovador.

Asimismo, los docentes percibían su desempeño profesional como una secuencia de rutinas escolares determinadas por el contexto. Las actividades del aula giraban sobre tres fases rutinarias: revisión de la tarea, presentaciones del profesor para introducir los tópicos nuevos, el desarrollo o inicio de la siguiente tarea. Dentro de estas actividades, el libro de texto jugaba un papel central en todas las lecciones observadas, ya que no sólo representaba la materia enseñada, sino que, además, determinaba la manera de ser presentada; convirtiéndose el docente en un simple traductor del libro de Matemática.

En cuanto a los valores particulares del área de las Matemáticas y, en general, de la cultura escolar, en esta investigación sobresalieron los siguientes:

la importancia de los exámenes y las notas;

la gran cantidad de temas que deben enseñarse en Matemática conduce al docente a "transmitir" sus conocimientos;

- poca habilidad en Matemática se cifra en un bajo nivel de inteligencia;

- $\quad$ los conceptos elementales no son responsabilidad del profesor de secundaria, sino de la enseñanza primaria;

la Matemática es de gran utilidad para cualquier disciplina, contribuye al desarrollo del pensamiento y representa una forma lógica de organizar y procesar la información.

- $\quad$ la actitud negativa hacia esta asignatura se explica por el perjuicio social. 
En este sentido, Escoffer y Prado (1993) plantearon la necesidad de que la formación docente genere una actitud comprensiva hacia la enseñanza sustentada en la creatividad, la iniciativa, el respeto mutuo, la solidaridad, la actitud empresarial, en el ejercicio de los derechos humanos y en el protagonismo de los estudiantes y las estudiantes; en fin, lo ínsito es formar docentes capaces de desempeñarse en un sistema educativo en constante cambio.

\section{Aproximación metodológica}

\section{Posición de la investigadora}

Este estudio se fundamentó en los criterios de calidad para la investigación cualitativa propuestos por Ivonna Lincoln (1995). En primera instancia, esta investigación no se hizo con el propósito de negar o confirmar hallazgos, teorías o postulados previos, sino que fue visto como un proceso de construcción del conocimiento generado a partir de la interacción con los profesores graduados y sus estudiantes; de tal forma que los resultados fueran significativos para ellos.

El segundo criterio fue la posicionalidad, entendida como una aproximación a la realidad plurisignificativa y heterogénea de los actores sociales; por ello se trató de rescatar sus vivencias, percepciones y significados a través de distintas técnicas, para demostrar que no existió una verdad absoluta, sino relativa a las particularidades de cada contexto.

La comunalidad fue concebida como una manera de servir a los propósitos del grupo de profesores de Matemática; transcendiendo la mera utilización de los participantes. Por lo tanto, se procuró corresponderles por el aporte de sus conocimientos y experiencias a esta investigación, mediante la interacción de la investigadora con los profesores para compartir, reflexionar y dialogar sobre los resultados y, de esta manera, encontrar alternativas pedagógicas que contribuyeran a mejorar los procesos de enseñanza y aprendizaje de la Matemática en su propio contexto.

Otros criterios de calidad fueron el rescate de las voces marginadas de los profesores y sus estudiantes por medio de diversas técnicas y la subjetividad crítica como un llamado de atención para establecer mi perspectiva dentro de la investigación, con el propósito de ampliar mi propia conciencia y producir transformaciones personales y sociales en los participantes, mediante un constante proceso dialógico y autoreflexivo. Finalmente, la discreción fue central puesto que implicó un respeto por la dignidad humana y por las relaciones interpersonales; por eso no se utilizaron los nombres reales de los actores.

\section{Perspectiva epistemológica}

En este artículo se reporta una investigación preliminar, específicamente un estudio de casos, que según Merriam (1988), es un diseño caracterizado por la descripción intensiva y holística, por ser particularista debido a su interés en un fenómeno específico. También se caracteriza por ser heurístico, puesto que permite explicar el transfondo de una situación, las razones de un problema; o bien, desarrollar la capacidad comprensiva e interpretativa del lector. Además, es inductivo porque las relaciones, los conceptos o hipótesis emergen de la examinación de los datos dentro de su contexto particular. De acuerdo con esta autora, las presunciones filosóficas del estudio de casos emanan de un paradigma cualitativo, debido al interés por el análisis inductivo, el proceso, la comprensión y la interpretación de una realidad múltiple surgida a partir de las interacciones y percepciones de los sujetos.

Por ello, esta experiencia investigativa fue un proceso que se construyó, reconstruyó o deconstruyó dentro del contexto educativo de la región educativa Huetar Atlántica, en el cual tanto los profesores como sus estudiantes construyeron sus propios significados e interpretaciones. 


\section{Técnicas}

Debido al poco tiempo para realizar el trabajo de campo -exclusivamente los meses de agosto y setiembre de 1996- éste es un primer acercamiento a la realidad educativa en el campo de la Matemática. La información se recolectó mediante la observación holística e interpretativa de la realidad de aula en tres tipos de colegios públicos: académico nocturno, académico diurno y técnico profesional ubicados en diferentes circuitos educativos de la región. Se realizaron tres observaciones no participantes a cada uno de los profesores, con el propósito de apreciar sus estrategias didácticas, sus interacciones con los estudiantes y las estudiantes y el ambiente afectivo dentro del aula. En este contexto, la investigadora asumió un rol pasivo: sólo observaba y anotaba; no interactuó en ningún momento con los estudiantes.

Por otra parte, se realizaron entrevistas en profundidad a cada uno de los profesores con la finalidad de ahondar en sus experiencias y significados sobre el convenio, la concepción de la Matemática, los cambios generados a partir de su formación profesional y el tratamiento metodológico de la Matemática. Tanto el número de entrevistas como de observaciones participantes estuvieron supeditadas a lo que Bogdan y Taylor (1992) llaman criterio de saturación, es decir, cuando la información ya no es novedosa y cuando las observaciones muestran la reiteración de algunos patrones dentro del espacio escolar.

Además, se empleó una técnica proyectiva basada en un instrumento de frases incompletas, elaborado con base en las expresiones manifestadas por varios estudiantes y profesores, distintos a los participantes de este estudio. Este instrumento se aplicó a tres estudiantes de cada docente graduado, con el propósito de rescatar sus creencias sobre la Matemática, específicamente sobre sus profesores, sus pruebas y su metodología. Lo primordial de esta técnica fueron las cuestiones de contenido, más que de procedimientos (Wittrock, 1989).

En cuanto a las estrategias de campo se recurrió a la negociación de la entrada en cada escenario dialogando, tanto con los profesores como con los directores de cada institución, sobre los propósitos y procedimientos de la investigación. Se fortaleció el "rapport" mediante un constante diálogo abierto con los profesores.

Finalmente, cabe destacar que se seleccionaron los cinco profesores graduados del Plan Regular de Formación Profesional porque lograron concluir su proceso de preparación docente de un grupo inicial constituido por casi treinta estudiantes. El perfil de estos profesores se resume en el siguiente cuadro.

\begin{tabular}{|c|c|c|c|c|c|c|c|}
\hline Sujetos & Edad & Sexo & $\begin{array}{l}\text { Años de } \\
\text { servicio }\end{array}$ & $\begin{array}{l}\text { Niveles } \\
\text { laborales }\end{array}$ & $\begin{array}{l}\text { Estudios } \\
\text { anteriores }\end{array}$ & Condición & $\begin{array}{l}\text { Tipo de } \\
\text { colegio }\end{array}$ \\
\hline Profesor 1 & 44 años & Masculino & 8 años & $10^{\circ}$ & $\begin{array}{l}\text { Ingeniería en } \\
\text { Construcción }\end{array}$ & Interino & $\begin{array}{l}\text { Académico } \\
\text { Nocturno }\end{array}$ \\
\hline Profesor 2 & 34 años & Masculino & 5 años & $8^{\circ}$ y $11^{\circ}$ & Computación & Propiedad & $\begin{array}{l}\text { Académico } \\
\text { Diurno }\end{array}$ \\
\hline Profesor 3 & 28 años & Masculino & 6 años & $8^{\circ}$ a $12^{\circ}$ & Computación & Propiedad & $\begin{array}{l}\text { Técnico } \\
\text { Profesional }\end{array}$ \\
\hline Profesor 4 & 32 años & Masculino & 12 años & $8^{\circ}$ y $9^{\circ}$ & Administración & Propiedad & $\begin{array}{l}\text { Académico } \\
\text { Diurno }\end{array}$ \\
\hline Profesor 5 & 26 años & Masculino & 7 años & $7^{\circ}$ y $8^{\circ}$ & Computación & Propiedad & $\begin{array}{l}\text { Académico } \\
\text { Diurno }\end{array}$ \\
\hline
\end{tabular}




\section{Credibilidad de la información}

Para lograr la credibilidad de la información se hizo una triangulación con base en la utilización de tres técnicas: la entrevista en profundidad para los profesores, la técnica proyectiva basada en un instrumento de frases incompletas para los estudiantes y las estudiantes y la observación participante.

Esta triangulación permitió una interpretación más precisa y clara de los resultados con base en la confrontación de la información obtenida de las notas de campo, de la transcripción de las entrevistas y de las expresiones dadas en el instrumento de frases incompletas. Como lo destacan Cook y Reichard (1986) si solo se dispone de una sola observación es imposible distinguir entre lo subjetivo y lo objetivo; sin embargo se pueden ajustar las observaciones por medio de instrumentos diferentes y desde diversos emplazamientos; entonces se logrará distinguir los componentes debidos al observador y a lo observado.

\section{Análisis e interpretación de los datos}

Las categorías de análisis o unidades de significación se construyeron a partir de la lectura, discusión y reflexión de la información. Además, se trató de descubrir en los datos los patrones de los profesores y sus estudiantes. Estas categorías, a su vez, se dividieron en subcategorías o propiedades descriptivas para una mayor especificación y para generar las siguientes conceptualizaciones.

\section{Categoria: Creencias sobre las Matemáticas}

Son las presunciones de la Matemática surgidas a partir de los sentimientos, pensamientos y convicciones expresados por los sujetos; los cuales se manifiestan de manera verbal y no verbal en el contexto del aula.

\section{Categoría: Articulación metodológica}

Es la aplicación de diversas estrategias y recursos didácticos para generar un proceso de reflexión, interacción y construcción de estrategias cognoscitivas entre los estudiantes y los participantes.

\section{Categoría: Concepción epistemológica}

Son los diversos procesos de apropiación del conocimiento matemático a partir de las experiencias cotidianas en el espacio escolar.

\section{Análisis de la información}

Para realizar el análisis fue necesario clasificar la información recolectada en categorías descriptivas, representadas por un código escrito en los márgenes. Posteriormente se hizo una síntesis de las manifestaciones verbales y no verbales en cada una de las categorías y subcategorías con el propósito de ordenar la información y de encontrar semejanzas o diferencias entre los actores sociales. Finalmente, se compararon y contrastaron las manifestaciones de los profesores con las de los estudiantes y las estudiantes y, en ciertos casos, con lo documentado.

Esto significó la reconstrucción detallada de las experiencias cotidianas y sus significados en el contexto del aula; cuya interpretación se hizo holísticamente para una mejor comprensión y se ilustrará con las expresiones de los sujetos participantes, tanto de profesores (P) como de los estudiantes y las estudiantes (E). Cada profesor se distinguió por un número específico: $\mathrm{P} 1, \ldots, \mathrm{P} 5$ y las expresiones empleadas fueron tomadas de las entrevistas (Ent.), el instrumento de frases incompletas (I.F.I.) y de las observaciones, las cuales se clasificaron numéricamente $01, \ldots, 04$. 


\section{Creencias sobre la Matemática}

\subsection{Creencias sobre el profesor}

Los profesores consideraron que no era suficiente tener algunos conocimientos elementales para enseñar Matemática; era fundamental una formación profesional para tener la capacidad de explicar cualquier contenido de diversas maneras.

P1 (Ent): "La formación debe ser con Matemáticas superiores, por lo tanto, si uno quiere ser profesor hay que estudiar (...) para saber mucho y enseñar poco."

Por su parte, los estudiantes y las estudiantes creían que el profesor ideal explicaba muy bien, con un vocabulario claro y entendible. Además debía reunir ciertas cualidades como: comprensivo, divertido, dinámico, agradable, respetuoso y comunicativo.

E. (I.F.I.): Creo que el profesor (a) ideal de Matemática debe ser "de fácil palabra y de mente abierta a nuestras peticiones y muy bien estudiado."

Se pudo apreciar que existió una imagen convencional del profesor de Matemática: conocedor de su campo. Sin embargo, los estudiantes y las estudiantes necesitaban algo más: un sujeto con mayor sensibilidad, quien se identificara más con sus intereses y necesidades. Como lo señala Saffie (1993) es necesario modificar las prácticas profesionales, centrándose no sólo en los conocimientos y en el hacer, sino prioritariamente en el área del ser.

\subsection{Creencias sobre la metodología}

Los profesores creían que en la enseñanza de la Matemática, no sólo se requería de un sólido dominio cognoscitivo, sino también saber como "transmitírselo" a sus estudiantes; puesto que no visualizaron la posibilidad de que éstos pudieran construir sus propios aprendizajes; desvalorizándose los conocimientos previos generados en otras experiencias. Asimismo, reconocieron la existencia de varios caminos, pero creyeron que debía emplear el método más simple y coherente.

P2 (Ent.): "Yo les digo a mis muchachos que no importa el método, siempre y cuando sea un procedimiento coherente; si yo fuera un profesor limitado que sólo me aprendí un método, le voy a tener miedo a otros métodos que no conozco, como le pasa a algunos."

Mientras que los estudiantes y las estudiantes creían que el profesor debía explicar muy bien, paso por paso, una y otra vez; ejemplificar y emplear recursos como los libros, la calculadora, la tiza de colores e instrumentos como la escuadra o el compás.

Se reafirmó, una vez más, las creencias tradicionales hacia la enseñanza de la Matemática, muy arraigadas en los participantes. Esto se justifica por ser el enfoque predominante en nuestro contexto educativo; en el cual se potencia la convergencia, cuyo eje central es el profesor; negándose los espacios para la exploración y la curiosidad. En este sentido, subraya Contreras (1994) que la manera más eficiente y eficaz de organizar la enseñanza de la Matemática ha sido usualmente el dividir los contenidos en pequeñas piezas, que pueden ser digeridas sistemáticamente por los estudiantes y las estudiantes.

\subsection{Creencias sobre las pruebas}

Los profesores creían que los estudiantes y las estudiantes obtenían bajos resultados en las pruebas porque no estudiaban lo suficiente, no tenían interés por aprender, se ausentaban y no realizaban los ejercicios de la práctica. Además, pensaban que las pruebas se resolvían con el razonamiento y la aplicación de operaciones.

P1, 01: "El poco interés y el ausentismo son los factores que explican el bajo rendimiento". Después expresó que las pruebas "son puro razonamiento, pensar un poquito y aplicar las operaciones fáciles.” (Décimo año).

Por su parte, los estudiantes y las estudiantes visualizaban sus aprendizajes en función de las pruebas; sólo les interesaba salir 
bien, por lo tanto, trataban de entender y estudiar con ese objetivo. Las pruebas no se percibieron como un reto para demostrar sus verdaderos aprendizajes, sino una manera de repetir cierta cantidad de materia, que en algunos casos es fijada por el número de páginas, y de resolver los ejercicios con los mismos procedimientos desarrollados por el profesor.

P4 (01): E: "A partir de qué página es el examen."

P: "Desde la 34."

E: “Toda la unidad!” (Octavo año).

El examen significaba para muchos angustia, inseguridad y temor, especialmente cuando sentían que no se habían preparado bien o les costaba mucho entender; por ello, algunos pensaron que tendrían que adivinar la respuesta correcta. Otros manifestaron sentir seguridad porque habían estudiado mucho y comprendido la materia.

E. (I.F.I.): Al escuchar la palabra Matemáticas siento "que me va a costar..."

Antes de hacer un examen de Matemática siento que "me lo voy a sacar malo".

Desde esta perspectiva, el examen se convirtió en un instrumento para controlar la cantidad de materia que debía ser aprendida de la misma forma, con solo un significado correcto, sin considerar las diferencias cognoscitivas de sus estudiantes.

\section{Articulación metodológica}

\subsection{La interacción docente y alumno}

Según los docentes, se debían propiciar relaciones de confianza y respeto para estimular los aprendizajes en los estudiantes.

Sin embargo, en diversos momentos se percibieron diferentes papeles del docente. Uno sobresaliente fue el del profesor modelo, quien debía ser imitado por sus estudiantes, siguiendo sus mismos pasos; reforzando el pensamiento convergente y menoscabando las potencialidades del estudiante y la estudiante para construir, a su manera, sus propios aprendizajes.
P1 (01): El profesor manda un estudiante a la pizarra y le dice: "Haga lo que acabo de hacer." (Décimo año).

En otros momentos fue el profesor autoritario, quien establecía las reglas del juego, quien controlaba a los estudiantes y las estudiantes para que realizaran el trabajo asignado; quien regañaba y amenazaba a los que molestaron, interrumpieron y no realizaron sus tareas.

P2 (02): "Aquí hay tres o cuatro personas que vienen a estorbar o molestar porque nada que ver (...). Si van a estar molestando mejor se van para la casa (...). Ahora vengo con los tacos de frente (...)". (Octavo año).

También resaltó el rol del profesor conocedor, él es quien sabía, explicaba, razonaba y, sobre todo, quien establecía los criterios de validez: lo correcto e incorrecto por su "status" de conocimiento; mientras que los estudiantes y las estudiantes no tenían los conocimientos básicos; consecuentemente su papel se centró en recibir ese conocimiento, escribirlo en sus cuadernos, limitarse a escuchar las explicaciones y a responder en forma individual o en coro; aunque algunos no respondían y otros sólo repetían lo dicho por sus compañeros.

P1 (02): P: "Desde dónde."

Responden en coro: "Desde -4 a 4".

El profesor hace la tabla, después traza los ejes; los estudiantes y las estudiantes lo observan y escriben en sus cuadernos. (Décimo año).

En fin, se puede afirmar que la dinámica dentro del contexto del aula tuvo como protagonista al profesor; limitándose los espacios para el diálogo, la reflexión y la interacción. Como lo indica Alfaro (1994) por la forma en que la educación ha sido concebi$\mathrm{da}$, se reduce a un sistema emisor y receptor; cuya comunicación tiene un sentido unívoco: hasta llegar al mismo significado.

\subsection{Clima afectivo}

En general, los profesores consideraban necesario mantener el orden, la disciplina y la atención de los estudiantes para desarrollar apropiadamente los contenidos curriculares. 
De ahí que, se percibieron diferentes climas en distintos grupos y momentos que se podrían ubicar en un continuum, desde uno rígido hasta el caótico. En el primer extremo se percibió un clima tenso, de poca empatía y de un gran distanciamiento entre el docente y el educando, posiblemente por su trato enérgico y exigente, puesto que no permitía ningún tipo de desórdenes y de interrupciones.

A un nivel intermedio se percibió un ambiente más agradable, tranquilo y menos tenso; quizás por el uso de expresiones cotidianas, jocosas y por un trato más familiar. En el otro extremo se sintió el predominio de un clima caótico, donde las estudiantes y los estudiantes no ejecutaban ninguna actividad de aprendizaje, lo que provocaba desorden: salían del aula, conversaban constantemente entre sí, se levantaban y desplazaban por el aula.

Sin embargo, hubo una situación presente en todas las observaciones realizadas a los cinco profesores: una cierta apatía hacia el aprendizaje evidenciada de muy diversas formas como: imitar sonidos, silbar, bostezar, expresar interjecciones o palabras que mostraban el cansancio y el hastío; o bien, hablaban de otros temas, hacían bromas o jugaban entre sí.

P4 (02): La estudiante que tenía calcomanías de "chiquita" en sus uñas no escribe, ni siquiera tiene su cuaderno abierto; a veces conversa con una compañera, otras veces se pone a cantar y luego expresó: "¡Ay, qué sueño!" (Octavo año).

Dentro de este clima los estudiantes y las estudiantes mostraron su afectividad hacia los otros compañeros de variadas formas, desde el uso de expresiones y bromas groseras hasta la manifestación de su apoyo con gestos y palabras.

Según los profesores, este clima se explicaba por la fuerte carga académica, las presiones y los problemas económicos y familiares. Además de estas particularidades contextuales, se podría pensar que esto fue un reflejo del rol pasivo del estudiante y la estudiante. Para cambiar este ambiente afectivo sería necesario integrar sus experiencias y conocimientos previos con el propósito de generar una motivación intrínseca y despertar su potencial heurístico. Como lo plantea claramente Aritzondo y colegas (1988) es preciso que los objetos de conocimiento sean acercados al alumno en su verdadera naturaleza y se constituyan en una verdadera toma de conciencia de las cosas y de los procedimientos.

\subsection{Estrategias didácticas}

Las observaciones en el contexto del aula mostraron una estrategia muy definida y semejante en cuatro de los profesores, quienes elaboraban los conceptos con sus propias palabras, sin recurrir a ningún texto; posteriormente lo explicaban varias veces con sus propios ejemplos y concluyeron con las prácticas.

P2 (Ent.): "Yo soy de los pocos que puedo llegar sin ningún cuadernito con la receta o un libro a dar clases (...). Yo sé lo que estoy haciendo (...). Yo hago las redacciones ahí mismo. Yo la tengo en mi interior."

Explícitamente, tres de los profesores reconocieron que siguieron el ejemplo de un profesor del convenio para definir esta metodología. Mientras que para otro, fue un profesor que le impartió clases en cuarto y quinto año de secundaria. Como lo confirma Tonucci (1988) se establece institucionalmente un círculo vicioso donde el profesor pasa de estudiante a profesor reproduciendo los modelos con los cuales aprendió.

P1 (Ent.): "Sinceramente este estilo que yo tengo en estos momentos es un ejemplo de (...). Él sabe las definiciones, las da con sus propias palabras (...) y los ejemplos y toda la teoría él nos la da. El libro es solo una guía para él, pero como profesional en su campo, él sabe mucho."

El otro profesor recurrió, según el contenido desarrollado, al libro para dar los conceptos; o bien, para asignar los ejercicios. Él consideró que su forma de enseñar no se 
basaba en un único modelo, sino que seleccionó lo mejor de cada uno de sus profesores, sumado a su experiencia y conocimiento.

En todos los casos se recurrió a la tarea para reafirmar lo enseñado, siguiendo los mismos procedimientos planteados por el profesor. También fue constante el interrogatorio acerca de los pasos, la aplicación de fórmulas y la sustitución de valores; pero ninguna se dirigió a estimular un conflicto cognitivo para la reflexión y la discusión. Sólo en una ocasión un profesor planteó una forma diferente para multiplicar y sumar.

P5 (02): ¿Cuál es el procedimiento que tengo que hacer? ¿Qué es lo primero?", los alumnos le responden simultáneamente. “QQuiénes están negativos? ¿Quiénes están positivos? ¿Cuál es el más grande de esos dos números? Vamos con la ley de signos (...)". (Sétimo año).

La ilustración de algunos conceptos, figuras geométricas o problemas con objetos, hechos cotidianos o con historias fue una estrategia muy frecuente.

P4 (04): El profesor resalta que siempre es importante hacer un dibujo para resolver el problema. Hace el dibujo de un edificio en la pizarra y va anotando los valores conforme lee el problema. (Noveno año).

Otra estrategia fue enviar a los estudiantes y las estudiantes a la pizarra para resolver un ejercicio, ya sea en forma voluntaria o escogido por el profesor; quienes se apoyaban en su cuaderno o examen; evidenciándose una gran inseguridad. Sólo, en pocas ocasiones, se observó que el estudiante resolvía el problema sin ningún texto. Asimismo, varias veces, el profesor no permitió la ayuda de otros compañeros; negándose la oportunidad de que éste construyera su propio aprendizaje interactuando con los otros sujetos. Como lo plantea Wittrock (1990) la interacción entre compañeros incrementa el desarrollo del razonamiento lógico mediante un proceso de reorganización cognitiva activa generada por el conflicto cognitivo.

P2 (02): La estudiante titubea, no sabe el resultado, entonces el profesor dice: "No oiga, no hay nadie ahí. Resuélvalo usted." (Octavo año).
Esta última situación se reafirmó, aún más, con el hecho de que esporádicamente tuvieron la oportunidad de trabajar grupalmente. Sólo en una ocasión se realizó un trabajo por grupos; pero mientras unos resolvían el ejercicio, otros conversaban o discutían entre sí y se destacaba uno dictando o dirigiendo a los otros compañeros; o bien, el profesor los guiaba con sus preguntas usuales.

Cabe resaltar que el error fue aceptado por el docente pero no se reflexionó o cuestionó acerca del por qué; siendo éste, junto con los aciertos, fundamentales para la construcción de sus aprendizajes.

P1 (Ent.): "Les digo hasta el profesor comete errores, yo también cometo errores; entonces cómo no van a cometerlos ustedes."

A excepción de uno, los profesores interpelaron constantemente a sus estudiantes cuando explicaban, llamándolos por sus nombres, como una forma de atraer su atención hacia la pizarra; siendo ésta el recurso didáctico más utilizado, donde los estudiantes y las estudiantes deben enfocar su vista y atención.

Finalmente, trataron de motivar las lecciones mediante repasos o indicando sus logros o fracasos. Sobresalió la manera muy particular de un profesor, quien recurría a palabras familiares, los chistes, refranes o parangones con hechos cotidianos para crear un clima más agradable y generar un gusto por la asignatura.

P2 (Ent.): "Yo creo que la educación debe ser como lo dijo Walt Disney: El prefería entretener tratando de enseñar que enseñar tratando de divertir (...), yo pienso que el aprendizaje no debe ser tan tortuoso."

\section{Concepción epistemológica}

\subsection{La imagen del objeto matemático}

Prevaleció una percepción rígida, sistemática, estructurada y mecánica de la Matemática, fortaleciéndose la imagen de objeto acabado y repetitivo, por lo tanto, no fue 
atractivo ni novedoso para los estudiantes y las estudiantes; pese a que los docentes la consideran una materia diferente: "no es memoria, sino razonamiento".

\section{P2, 01: "Vamos a trabajar con recetas de cocina".}

Por su parte, los estudiantes y las estudiantes la percibían como algo útil para el futuro, para desenvolverse mejor en la vida diaria, para aprender cosas nuevas; pero no manifestaron que fuese realmente significativa para sus vidas. Más bien se percibió como un conocimiento externo, objetivo y socialmente valorado, que como algo aprehendido por sí mismo.

E (I.F.I.): Pienso que la Matemática es útil para "la vida diaria."

También se evidenció una imagen positiva de la Matemática cuando se entendía el tema; o bien, era difícil cuando: no se comprendía, no se prestaba atención, se faltaba a clases, había muchas operaciones por resolver o el profesor no explicaba bien o lo hacía muy rápido.

E. (I.F.I.): Al escuchar la palabra Matemática siento "que muchos números pasan por mi cabeza.”

Según los profesores, a los estudiantes no les gustaba la Matemática y sentían temor cuando debían realizar muchas operaciones o no comprendían el lenguaje técnico. Dos de los docentes creían que la Matemática no es para todos.

Una vez más se reafirmó esa imagen compleja, sublime e inaccesible; que implica para muchos un gran esfuerzo y dedicación para aprender lo básico y salir bien en los exámenes.

\subsection{La apropiación del conocimiento matemático}

Según los profesores, sus estudiantes se apropiaban del conocimiento matemático cuando se aprendían de memoria los conceptos, leyes, propiedades y aplicaban los procedimientos desarrollados por ellos, paso a paso, en las prácticas.

P4 (04): El profesor sigue explicando la operación: "Tienen que aprendérselo de memoria para hacerlo". (Noveno año).

Los ejes centrales del aprendizaje matemático eran: prestar atención, razonar y practicar; así fue como ellos aprendieron.

P2 (Ent.): “(...) sólo hay que ponerle atención al profesor, practicar lo que él explicaba y analizar un poco. Quizás más que todo es análisis."

A juicio de los profesores, sus estudiantes no aprendían debido a la falta de disciplina para el estudio; además, sólo escribían, no prestaban atención y no habían tenido una preparación apropiada en años anteriores, especialmente en la escuela; de tal forma que el dominio de los conceptos básico no fue responsabilidad de ellos, sino del maestro.

Por su parte, los estudiantes y las estudiantes, consideraron que la apropiación del conocimiento matemático implicaba mucho estudio, interés y esfuerzo; especialmente si tenían dificultades para aprender. Asimismo, pensaban que el profesor debía explicarles claramente, varias veces y con un lenguaje sencillo.

E. (I.F.I.): Mis notas en Matemáticas se deben a que "el profesor explica bien y me esfuerzo por entender."

Tanto en las percepciones de los profesores como de sus estudiantes, hubo coincidencias que reafirmaban el aprendizaje matemático como un proceso automático, estático, memorístico, repetitivo y rígido.

\section{Limitaciones del estudio}

La gran limitación de esta experiencia investigativa fue el tiempo, puesto que el trabajo de campo se realizó en dos meses; por consiguiente no hubo espacios para la interacción de la investigadora con los estudiantes y las estudiantes en su ambiente escolar. 
Además, no se logró profundizar, mediante entrevistas, en las percepciones de los estudiantes y las estudiantes sobre sus propios aprendizajes y creencias acerca de la Matemática. Tampoco se rescataron las voces y presunciones de los padres de familia de los estudiantes y las estudiantes que interactuaron con los profesores graduados del convenio. Finalmente, podría señalarse la falta de profundización, en algunos significados, de las experiencias del docente en el convenio y el trabajo de aula.

\section{Consideraciones finales}

En primera instancia, cabe resaltar que la formación docente influyó en los cinco profesores graduados, especialmente en la solidez de sus conocimientos matemáticos; generando en ellos una gran seguridad y confianza para desarrollar los contenidos curriculares; ya que antes de ingresar al convenio se apoyaban, casi exclusivamente, en el libro de texto. Sin embargo, no se apreció en el trabajo de aula una diversidad en los tratamientos metodológicos, pues se centraron en dar los conceptos, explicarlos, ejemplificarlos y proporcionar una práctica, que los estudiantes resolvían siguiendo el mismo procedimiento.

Sumado a lo anterior, sus articulaciones metodológicas no se generaron de un proceso de autoreflexión y concienciación, puesto que para ellos su forma de enseñar era el reflejo de algún profesor de Matemática del convenio o de secundaria; fortaleciéndose el ciclo de "cómo se me enseñó, así yo enseño". Además, esta relación de espejo se fortaleció por el hecho de que las materias de pedagogía no fueron significativas, al no estar contextualizadas a su realidad y, para algunos, no tenían ninguna utilidad para su trabajo de aula.

También sus articulaciones metodológicas fueron muy tradicionales, centradas en el docente, como el dueño del conocimiento $\mathrm{y}$, por lo tanto, es quien explica, razona, ejemplifica, resuelve y, sobre todo, establece lo correcto e incorrecto; lo que conlleva al fortalecimiento del conocimiento convergente y objetivo de la Matemática.

Dentro de esta perspectiva, el estudiante y la estudiante tuvo un rol pasivo: receptor en serie de ese conocimiento, se aprendió del mismo modo y con los mismos significados. Consecuentemente, no existieron espacios para que los y las estudiantes reflexionaran, experimentaran, compararan y desarrollaran más libremente sus estructuras cognoscitivas, en niveles más complejos, para construir sus propios conocimientos, tanto con sus aciertos como con sus errores y sus particulares significados.

Por otra parte, esta articulación metodológica no ha generado un cambio significativo en las creencias y, por ende, en las actitudes de los estudiantes y las estudiantes hacia la Matemática, puesto que privó la imagen de un área disciplinaria, compleja y abstracta que demandaba un gran esfuerzo para apropiarse de un conocimiento mínimo.

En este sentido, sería muy importante buscar otras alternativas, generadoras de ese cambio significativo hacia la enseñanza de la Matemática. Se podría pensar en la necesidad de crear espacios lúdicos, la ejecución de actividades en diferentes contextos para el desarrollo del potencial heurístico; la proporción de materiales didácticos que estimulen el razonamiento $y$, específicamente, configuren una concepción más concreta y cercana a sus vivencias, integrando los programas curriculares con las expectativas, intereses y necesidades de los estudiantes y las estudiantes, resaltando los propósitos para que tomen conciencia de sus propios aprendizajes.

Finalmente, es recomendable el fortalecimiento de un clima más propicio, retador, estimulante y libre para el aprendizaje, a partir de una interacción más dialéctica con los otros compañeros y menos vertical con el docente y cuyo principal motor de esta dinámica en el contexto de aula sea el estudiante y la estudiante. 


\section{Referencias bibliográficas}

Alfaro, G. "El educador como aprendiz". Revista de la Universidad de Costa Rica: Educación. № 18 (1). San José: Editorial de la Universidad de Costa Rica, 1994.

Apraiz, J., Alloza, J.J., Vicario, J.L. et al. "Capacitación matemática en el ciclo medio. Su evaluación. En Temas actuales sobre Psicología y Didáctica. Madrid: Narcea S.A. Ediciones, 1988.

Aritzondo, M.J., Gómez, J.F. y Huarte, F. "La importancia de la comprensión de la finalidad de las actividades escolares en el proceso de conceptualización: una experiencia con niños de 11-12 años". En Temas actuales de Psicopedagogía y Didáctica. Madrid: Narcea S.A. Ediciones, 1988.

CONARE. Necesidades de Formación de Profesores en las asignaturas de Español, Estudios Sociales, Ciencias, Matemática e Inglés para los próximos años. Costa Rica: CONARE, 1991.

Contreras, I. "El rol del profesor de Matemáticas en la educación secundaria: Un referente teórico para su estudio". Revista de la Universidad de Costa Rica: Educación. San José: Editorial de la Universidad de Costa Rica, No 18 (1), 1994.

Contreras, I. "El quehacer diario del profesor de Matemática en la Educación Secundaria: algunos resultados de su estudio". Revista de la Universidad de Costa Rica: Educación. San José: Editorial de la Universidad de Costa Rica, Vol. 19 (1), 1995.

Cook, T.D. y Reichardt, Ch.S. Métodos cualitativos y cuantitativos en investigación evaluativa. Madrid: Ediciones Morata S.A., 1986.
Escoffer, M. y Prado, M. "Formación docente: Apuntando al profesionalismo.” Revista de Chile: Educación. No 211, 1993.

Kerlinger, F. Investigación del comportamiento. México: Editorial McGraw-Hill, 1994.

Lincoln, I. "Standards para la investigación cualitativa: Criterios emergentes de calidad en la investigación cualitativa interpretativa". San Francisco: Ponencia presentada en Programa de la Asociación Americana de Investigación educativa, 1995.

MEP. El reto del tercer milenio. Una propuesta de un proyecto educativo hacia el 2005 (EDU2005). San José: MEP, 1995.

Merriam, Sh. Case study research in education. A qualitative approach. San Francisco: Jossey-Bass Publishers, 1988.

Pereda, L. y Mazarredo, I. "Rendimiento académico en Matemáticas, por ciclos (inicial, medio y superior) y por modelos educativos A y D.". Temas actuales sobre psicología y didáctica. Madrid: Narcea S.A. Ediciones, 1988.

PROMECE. Evaluación del Convenio M.E.P.Universidades Estatales para la formación de docentes. PROMECE: Costa Rica, 1995.

Rechc, J., Hartzell, J. y Stephens, L. "Comparisons of Mathematical competencies and attitudes of elementary education mayors with established norms of a general college population". Revista de la Universidad de Nebraska. Omaha: School Science and Mathematics, Volumen 93 (3), March, 1993.

Saffie, N. "Problemática y sugerencias metológicas. Cómo orientar al adolescente 
de bajo rendimiento escolar". Chile: Revista de educación, $\mathrm{N}^{\circ}$ 213, 1993.

Taylor,S. y Bogdan, R. Introducción a los métodos cualitativos de investigación. Argentina: Editorial Paidós, 1992.

Tonucci, F. "Enseñanza-aprendizaje: una antigua relación que hoy tenemos que verificar". En Temas actuales sobre
Psicopedagogía y Didáctica. Madrid: Narcea S.A., 1988.

Wittrock, M. La investigación de la enseñanza II: Métodos cualitativos y de observación. España: Ediciones Paidós, 1989.

Wittrock, M. La investigación de la enseñanza III: Profesores y Alumnos. España: Ediciones Paidós, 1990. 